\title{
Design of Mobile Robot Hand-Held Wireless Control Terminal System
}

\author{
Chong Zhao \\ School of Science \\ Xijing University \\ Xi'an, China, 710123 \\ zhaochong@xijing.edu.cn
}

\author{
Ning Wang and Min Ji \\ School of Science \\ Xijing University \\ Xi’an, China, 710123
}

\begin{abstract}
To meet the demand of wireless remote control to intelligent vehicles and mobile robots, a hand-held wireless control terminal system based on touch is proposed in this paper. The system hardware uses ATmega64A Single-chip Microcomputer(SCM) as the main controller, integrating with high-speed wireless transceiver, 2.8 inch LCD with touch panel, font chip and SD card. The system software is porting hardware drive and designing human-computer interface. The experimental test shows that the system is easy to hand-held, work properly with reliable performance, smart touch and beautiful appearance.
\end{abstract} robots

Keywords-ATmega64; wireless remote control; touch control;

\section{INTRODUCTION}

For the control of intelligent vehicle and mobile robot, the mobile control terminal is an important part of monitor and the robot movement control. By the real-time monitoring of robots, it can access the motion state, record the sensor data, check the trend of parameter change and deal with alarm, etc. Generally, mobile control terminals are composed of mobile computing platform, wireless transceiver module and upper computer monitoring software. There are usually three options for choosing: the first, Personal computer (PC) or Programmable Logic Controller (PLC), wireless transceiver and upper computer software[1][2][3]; the second, Smartphone, wireless transceiver and mobile phone software[4][5]; the third, Embedded Microcontroller Unit (MCU), wireless transceiver and embedded software[6][7]. For the first scheme, PC or PLC have the rich extension interfaces, but the large volume and heavy quality is bad for the mobile control application. For the second scheme, Smartphone are portable for hand-holding and flexible for touching, but the application is limited with the difficulty of extend peripherals. For the third scheme, MCU can connect multiple peripherals and sensors with the rich peripherals. The system can be extend and designed flexibility for the functional requirements.

In conclusion, the third scheme is better. It fuses the advantages of the first two schemes and combines wireless technology with touch technology to make it has the widely application of wireless touch. By the touch screen technology, users operate robot easily with their fingers touch the display screen. This new attractive interactive device makes humancomputer interaction become more simple and convenient. It not only freed people from the keyboard and mouse, but also makes people to control the mobile device conveniently[8], so that the functions of touch input and rich graphics display could be realized easily. The wireless data transmission with $2.4 \mathrm{GHz}$ high-speed wireless transceiver terminal makes the control of intelligent vehicle and mobile robot become more conveniently[9].

\section{SYSTEM HARDWARE DESIGN}

The system hardware consists of the ATmega64A SCM, the high-speed wireless transceivernRF24L01+, the 2.8 inch LCD with touch panel, the font chip GT20L16S1Yand SD card, etc. The hardware structure diagram of the system is shown in Fig. 1. The core part of the system is the SCM minimum system with ATmega64A.

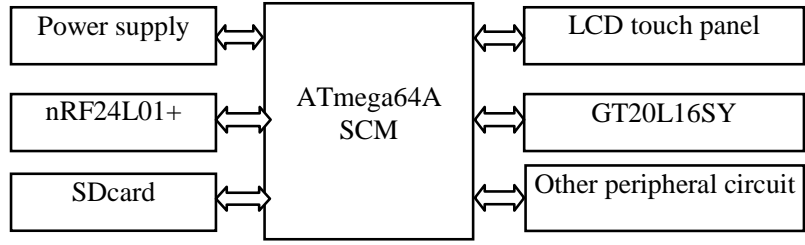

Fig. 1. The structure diagram of system hardware

\section{A. ATmega64ASCM and GT20L16S1Y font chip}

The system uses embedded microprocessor Atmega64A of Atmel. The Atmega64A has high performance and low power consumption, most instruction execution time is less than single clock cycle. When it working at $16 \mathrm{MHz}$, the performance can reach 16MIPS. It has 8 32-bit generalpurpose registers, the multiplier just needs two clock cycles, On-chip FLASH ROM 64KB, EEPROM2KB,SRAM4KB,2 USART, 1 double speed SPI interfaces (working at $16 \mathrm{MHz}$, the SCK clock can reach $8 \mathrm{MHz}$ ), 1 IIC interface, watchdog timer, etc. So, Atmega64A can meet the design requirements of handheld wireless application system.

The font chip GT20L16S1Y contains the standard Chinese font with 15 x 16 dot matrix. It has GB2312 national standard simplified Chinese characters, contains 6763 Chinese characters and 6 sets of ASCII characters $(5 \times 7 \sim 8 \times 16$ dot matrix). By the character code, the users can calculate the address in the chip with the method provided by the chip, and from this address the dot matrix information can be read. 


\section{B. The interface circuit design of LCD touch screen}

The LCD touch screen interface circuit consists of the touch screen interface circuit and the LCD interface circuit. The touch screen interface circuit is shown in Fig. 2. The touch screen interface circuit connects to the embedded microprocessor by the SPI bus via of TSC2046. With the collection, calculate and filter[10] of real-time touch screen data, it get the user's touch behavior and the touch coordinate location data. The LCD interface circuit connect to the embedded microprocessor via the 8-bit parallel interface. The 74HC245 drives LCD panel [11] to achieve graphic image rendering and interface display.

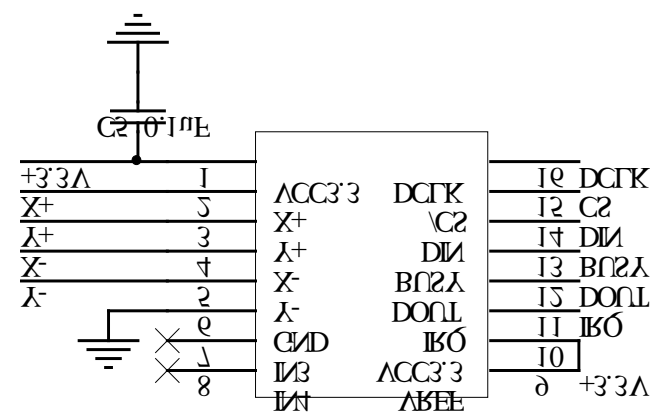

Fig. 2. The diagram of touch screen interface circuit

\section{The interface circuit design of font chip and SD card}

In view of the low Flash ROM storage capacity of ATmega64A,the interface circuit of font chip and SD card be design for the large capacity data storage and character display. The interface circuits are shown in Fig. 3 and 4 respectively.

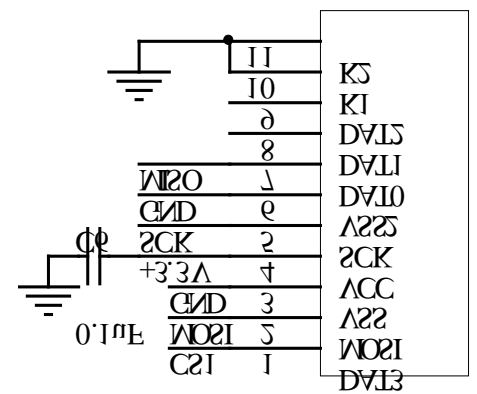

Fig. 3. The diagram of SD card interface circuit

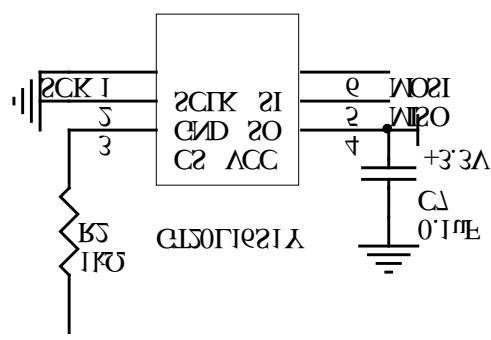

Fig. 4. The diagram of font interface circuit

\section{Wireless module design}

we selectnRF24L01+ for this module, a single chip radio frequency(RF) transceiver is widely used which is produced by the Nordic companies. The nRF24L01+ working at 2.4 $\mathrm{GHz}$ frequencies, the data transfer rate can reach $2 \mathrm{MB} / \mathrm{s}$. It

has the function of automatic response, automatic retransmission and CRC check. It also has 125 optional work channel, and the short channel switching time can be used for the frequency modulation. The 5V level of SCM be converted to $3.3 \mathrm{~V}$ level and connect with the SPI interface of nRF24L01+.The interface circuit of wireless transceiver is shown in Fig. 5.

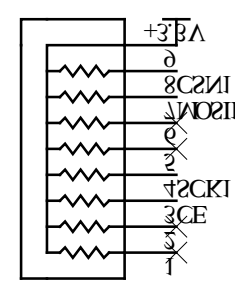

BJ J JOK2

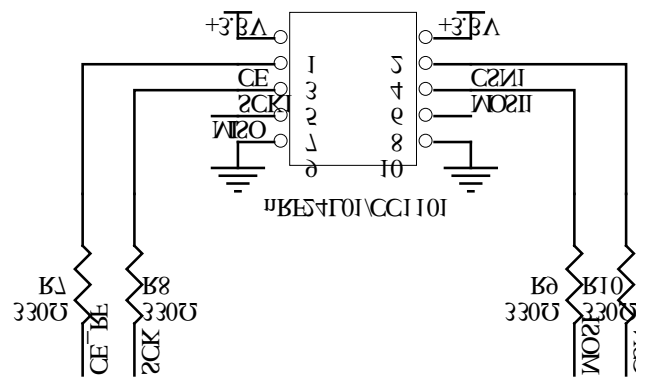

Fig. 5. The interface circuit of wireless transceiver

\section{E. The design of system power supply}

The system has two types of power supply, USB and battery $(6-9 \mathrm{v})$. The SCM needs $5 \mathrm{~V}$, others need $3.3 \mathrm{~V}$, and the maximum system current is less than $300 \mathrm{~mA}$. We chose the power chip AMS1117-5.0 to provide 5V, and the ASM11173.3 to provide $3.3 \mathrm{~V}$.

\section{SYSTEM SOFTWARE DESIGN}

The AVR Studio and Win AVR are used for system development, and system software adopts modular design. On the hardware platform, the hardware driver is transplanted, and the graphic image library(GIL), character display library(CDL), touch keypad(TK), wireless data processing library(WDPL) and human-computer interaction interface program(HCIIP) are written. The software structure diagram is shown in Fig. 6.

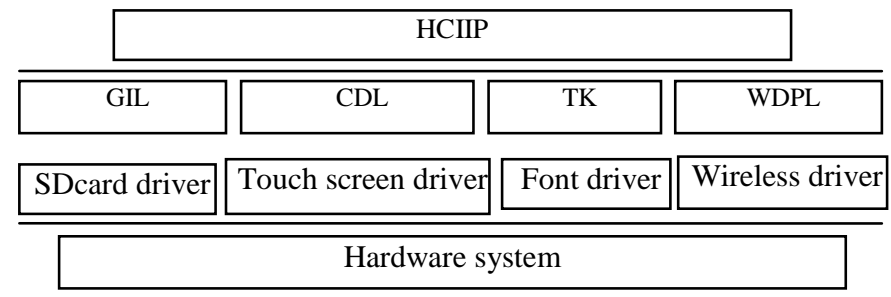

Fig. 6. The software structure diagram

System software is divided into four steps: (1) Initialize SCM and hardware circuit; (2)Transfer the graphics image library to build graphical window; (3)Display the main interface; (4)Wait for the button operation, then executing function according to user's choice. The software process diagram is shown in Fig. 7. 


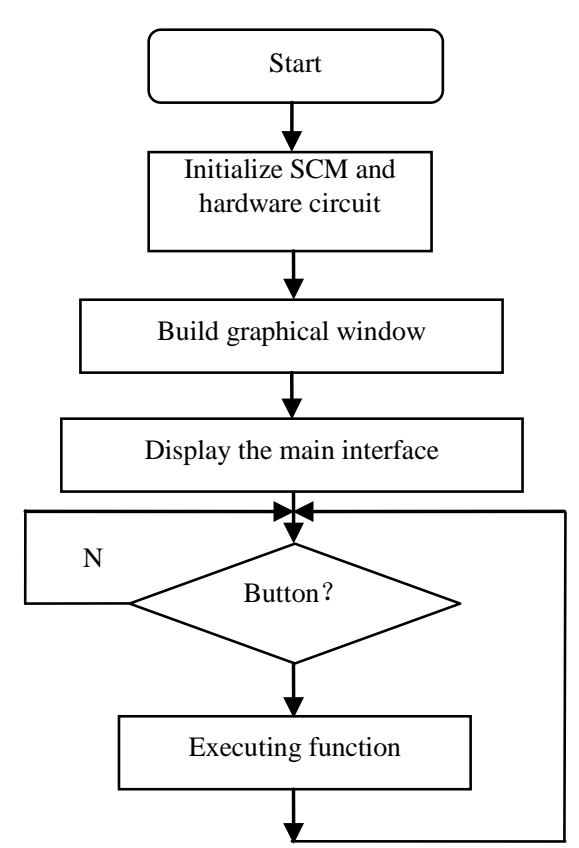

Fig. 7. The diagram of software process

The main program code:

int main(void)

\{

Port_Init(); // Port initiation

Uart_Init(); // Serial port initiation

ILI9325_Initial();//LCD initiation

ClearScreen(Black);

SetfbRGB(Green,Black);

Touch_Init(); // Touch screen initiation

SD_Init(); //SD card port initiation

SPI_GT20L16_Init(0); //Font initiation

get_first_data_from_ROM();

uart_puts ("GT20L16_Init High Speed OK!n");

Wireless_Init();//Wireless module initiation

Cleararea(13,13,147,226,Black);

CreateWindow();//Build graphical window

DisplayPanel(); //Generate main interface

Set_Linedis(2); // Sets the character line spacing

Set_Columedis(0);

while(1) /////////////Main

cyclic///////////////////////////

\{

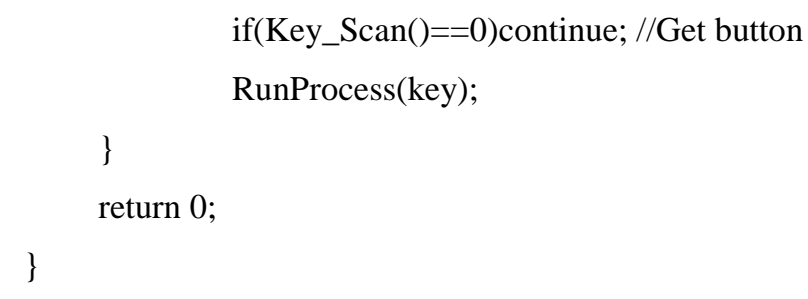

\section{SYSTEM TEST AND RESULTS}

There are two aspects of system testing: the test of wireless data transceiver distance and the test of touch response time. At the system clock $12 \mathrm{MHz}$, the experimental results are as follows:

(1) In the open field, the communication distance can reach $100 \mathrm{~m}$ with the data transmission rate $250 \mathrm{~Kb} / \mathrm{s}$ and the transmit power $0 \mathrm{dBm}$. In the house or $2.4 \mathrm{GHz}$ coverage areas, the communication distance usually around $50 \mathrm{~m}$ with the same data transmission rate and the same transmit power.

(2) With 200 touch tests, the following data was obtained: the minimum response time is $8.2 \mathrm{~ms}$, the maximum response time is $25.6 \mathrm{~ms}$, and the average response time is $14.7 \mathrm{~ms}$.

The experimental data shows that the system with the characteristics of reliable communication, flexible touch and convenient movement. In practical application, the control requirements of intelligent vehicles and mobile robot can be satisfied, and the better results can be obtained.

\section{REFERENCES}

[1] Yin, Q., Zeng, Y. H., Gao, Q. J, et al. Research and design of a kind of universal remote terminal for mobile robot [J]. Machinery Design \& Manufacture, 2012(4): 168-170.

[2] Hou, B. M., Feng, J. X. Du, F, et al. Design and implement of teleoperation interface system for mobile robot [J]. Modern Electronics Technique, 2009(10): 34-35.

[3] Su. Y., Pan. F. Remote Control System of Harrow Machine Based on Kingview and PLC [J].Industrial Instrumentation \& Automation, 2004(2): 53-55.

[4] Jiang. Y. L. Remote Control System Based on Android Intelligent Terminal [J]. Application of Electronic Technique, 2012, 38(8): 136-139.

[5] Fu, Q., Ren, F. H. Design of Remote Control Vehicle Based on Bluetooth of Mobile Phone [J]. Modern Computer, 2011(31): 77-79.

[6] Jiang, X. N., Huang, Y. H., Wang, X. P. Design and Implementation of Unified Embedded Infrared Remote Controllers [J]. Computer Engineering and Design, 2011, 32(1): 125-128.

[7] Yuan, J. J. Research on Remote Control of Embedded Mobile Robot Based on ARM9 [D]. Tianjin: Tianjin University of Technology, 2006.

[8] Wang, Y. Technology Market Trend of Touch Button \& Panel [J]. Electronic Engineering \& Product World, 2010(7): 12-19.

[9] Liu, J., Chen, Z. P., Li, Q. L. Design of Wireless Communication System Based on nRF24L01 [J]. Journal of Tianjin University of Technology, 2007, 23(3): 38-40.

[10] Song, X. R., Cai, Z. Y., Duan, Q. Q. Data Processing Algorithm of Touch Screen [J]. Computer Engineering, 2008, 34(23): 255-257.

[11] $\mathrm{Wu}, \mathrm{Q}$. Design and implementation of touch screen machine interface system based on AVR microcontroller [J]. Computer Knowledge and Technology, 2013, 9(23): 5357-5359. 